\title{
Ropivacaine induces cell cycle arrest in the G0/G1 phase and apoptosis of PC12 cells via inhibiting mitochondrial STAT3 translocation
}

\section{Lian Zeng}

Xiangyang No 1 People's Hospital Affiliated to Hubei University of Medicine

\section{Zhen Zhang}

Xiangyang No 1 People's Hospital Affiliated to Hubei University of Medicine

Fuyu Zhang

Xiangyang No 1 People's Hospital Affiliated to Hubei University of Medicine

Huaxian Chen

Xiangyang No 1 People's Hospital Affiliated to Hubei University of Medicine

\section{Ying Wang}

Xiangyang No 1 People's Hospital Affiliated to Hubei University of Medicine

\section{Xudong Ding}

Xiangyang No 1 People's Hospital Affiliated to Hubei University of Medicine

Huiyu Luo ( $\nabla$ 603983267@qq.com )

Xiangyang No 1 People's Hospital Affiliated to Hubei University of Medicine https://orcid.org/00000003-2126-223X

\section{Research Article}

Keywords: ropivacaine, neurotoxicity, STAT3, mitoSTAT3, PC12 cells, spinal cord

Posted Date: May 7th, 2021

DOI: https://doi.org/10.21203/rs.3.rs-486893/v1

License: (c) (1) This work is licensed under a Creative Commons Attribution 4.0 International License. Read Full License

Version of Record: A version of this preprint was published at Inflammation on August 20th, 2021. See the published version at https://doi.org/10.1007/s10753-021-01508-w. 


\section{Abstract}

STAT3 has neuroprotective effect via non-canonical activation and mitochondrial translocation, but its effects on ropivacaine-induced neurotoxicity remain unclear. Our previous study revealed that apoptosis was an important mechanism of ropivacaine-induced neurotoxicity, this study is to illustrate the relationship between STAT3 with ropivacaine-induced apoptosis. Those results showed that ropivacaine treatment decreased cell viability, induced cell cycle arrest in the G0/G1 phase, apoptosis, oxidative stress, and mitochondrial dysfunction in PC12 cells. Besides, ropivacaine decreased the phosphorylated levels of STAT3 at Ser727 and downregulated the expression of STAT3 upstream gene IL-6. The mitochondrial translocation of STAT3 was also hindered by ropivacaine. To further illustrate the connection of STAT3 protein structure with ropivacaine, the autodock-vina was used to examine the interaction between STAT3 and ropivacaine, and the results showed that ropivacaine could bind to STAT3's proline site and other sites. In addition, the activator and inhibitor of mitoSTAT3 translocation were used to demonstrate it was involved in ropivacaine-induced apoptosis, the results showed that enhancing the mitochondrial STAT3 translocation could prevent ropivacaine-induced apoptosis. Finally, the expression of p-STAT3 and the levels of apoptosis in the spinal cord were also detected, the results were consistent with the cell experiment, ropivacaine decreased the expression of p-STAT3 protein and increased the levels of apoptosis in the spinal cord. We demonstrated that ropivacaine induced apoptosis by inhibiting the phosphorylation of STAT3 at Ser727 and the mitochondrial STAT3 translocation. This effect was reversed by the activation of the mitochondrial STAT3 translocation.

\section{Introduction}

Ropivacaine, as an amide local anesthetic, has been widely used in clinical practice. Because of the longlasting and stable anesthetic effects, ropivacaine is mainly used for pain management and regional anesthesia, which has the characteristic of sensorimotor separation ${ }^{[1]}$. However, some studies have shown that ropivacaine has cytotoxicity ${ }^{[2,3]}$, including muscle toxicity, neurotoxicity, and cardiotoxicity. In recent years, increasing attention has been paid because its neurotoxicity is directly related to the recovery of patients after surgery ${ }^{[4]}$, but the mechanism remains unclear. In decades, a growing body of evidence has revealed that apoptosis plays an important role in the neurotoxicity of ropivacaine ${ }^{[5-7]}$, our previous study demonstrated that ropivacaine induced apoptosis of PC12 cells via Fas / Fasl mediated exogenous apoptotic pathway ${ }^{[8]}$.

The signal transducer and activator of transcription 3 (STAT3), as a transcriptional enhancer of acutephase genes activated by IL-6 ${ }^{[9]}$, has been demonstrated to be involved in many biological activities including apoptosis, cell cycle progression, cell migration, and angiogenesis ${ }^{[10-12]}$. Many studies indicate that the role of STAT3 in inhibiting apoptosis depends on mitochondrial STAT3 (mitoSTAT3)

translocation ${ }^{[13,14]}$, and the transduction of downstream signals initiated by STAT3 depends on the phosphorylation of specific amino acids, the most representative of which is the tyrosine705 (Tyr705) or serine727 (Ser727) phosphorylation ${ }^{[9]}$. The Ser727 phosphorylation of STAT3 could enhance mitoSTAT3 
translocation ${ }^{[14]}$. The anti-apoptosis of mitoSTAT3 is involved in improving damaged mitochondrial functions including regulating the mitochondrial electron transport chain (ETC) activity and increasing the mitochondrial membrane potential $(\psi \Delta \mathrm{m})$ to elevate ATP production ${ }^{[15]}$. MitoSTAT3 has been demonstrated to have a protective role in many diseases including ischemia ${ }^{[16,17]}$, immune system disease ${ }^{[18]}$, and other diseases ${ }^{[19]}$. However, there is no study involving the relationship between mitoSTAT3 with ropivacaine-induced neurotoxicity.

In the present study, our results showed that ropivacaine inhibited the Ser727 phosphorylation of STAT3 and mitoSTAT3 translocation, which might contribute to the neurotoxicity of ropivacaine in PC12 cells and the spinal cord including decreasing cell viability, inducing cell cycle arrest in the G0/G1 phase, apoptosis, oxidative stress, and mitochondrial dysfunction.

\section{Materials And Methods}

Rat pheochromocytoma cell line (PC12) was purchased from the Cell Bank of Shanghai Institute of Chinese Academy of Sciences. These cells were maintained in Dulbecco's modified Eagle's medium (DMEM) with $10 \%$ fetal bovine serum (FBS) and 1\% penicillin/streptomycin. The cell counting kit-8 (CCK8) from Biosharp (Shanghai, China), lactate dehydrogenase (LDH), superoxide dismutase (SOD), and malondialdehyde (MDA) test kits from wanleibio (Shenyang, China), reactive oxygen species (ROS), Mitochondrial membrane potential test kits (JC-1) and Mitochondrial protein extraction kits purchased from KeyGEN BioTECH (Jiangsu, China), primary antibodies against CyclinE1, CDK4, p21, Bax, Bcl-2 from Proteintech (Wuhan, China), Caspase-3, STAT3, p-STAT3, and COXIV from Wanleibio (Shenyang, China) were used in the present study.

\section{Cell cultured and drug administration}

PC12 cells were grown in the environment containing $10 \% \mathrm{CO}^{2}$ at $37^{\circ} \mathrm{C}$. When the confluence of the cells reached $70-80 \%, 0.25 \%$ trypsin with EDTA was used to digest the cells, which were then harvested after centrifugation. These cells were divided into the control group and different ropivacaine groups $(0.5 \mathrm{mM}, 1$ $\mathrm{mM}, 2 \mathrm{mM}$ ). After incubation with serum-free high-glucose DMEM for $12 \mathrm{~h}$, ropivacaine (Hengrui medicine, Jiangsu, China) was added to the medium, followed by incubation for $24 \mathrm{~h}$.

\section{Cell viability detection}

PC12 cells were seeded in a 96-well plate at a density of $5 \times 10^{3}$ cells/well and then treated with PBS or various concentrations of ropivacaine $(0.5 \mathrm{mM}, 1 \mathrm{mM}, 2 \mathrm{mM}, 4 \mathrm{mM}, 8 \mathrm{mM})$ in $100 \mu$ l of medium for the indicated times. There were six wells in each group. After incubation for $24 \mathrm{~h}, 10 \mu \mathrm{l}$ of CCK-8 solution was added to each well, followed by incubation for $2 \mathrm{~h}$ at $37^{\circ} \mathrm{C}$. The absorbance was measured in a microplate reader (Biotek, Winooski, VT, USA) at a wavelength of $450 \mathrm{~nm}$.

\section{ROS, SOD, MDA, and LDH determination}


The cells and cell supernatant were collected to measure the levels of SOD, MDA, and LDH with corresponding kits according to the manufactures' instructions. Then, PC12 cells were seeded in a 12-well plate at a density of $5 \times 10^{4}$ cells/well. After treatment with different concentrations of ropivacaine, cells were incubated with dihydroethidine (DHE) for $60 \mathrm{~min}$ in dark following the instructions of ROS assay kit. Subsequently, the fluoresce intensity was observed under a fluorescence microscope (Olympus, Japan) and the average optical density was measured by ImageJ software.

\section{Mitochondrial and nuclear DNA ratio (mtDNA / nDNA)}

The DNAzol ${ }^{T M}$ Reagent (Invitrogen ${ }^{T M}$, USA) was used to extract the genomic DNA according to the manufacturer's instructions. DNA was quantified with NanoPhotometer (Implen, Germany). The ratio of mtDNA / nDNA was measured by real-time PCR. The amplification of mitochondrial cytochrome $c$ oxidase subunit II (COII, mitochondrial-encoded gene) and $\beta$-actin (nuclear-encoded gene) was done with Real-time PCR. The primers used were as follows: COIl: 5'-TGAGCCATCCCTTCACTAGG-3'(sense) and 5'TGAGCCGCAAATTTCA GAG-3' (anti-sense); $\beta$-actin: 5'-CTGCTCTTTCCCAGATGAGG-3'(sense) and 5CCACAGCA CTGTAGGGGTTT-3' (anti-sense).

\section{Mitochondrial staining}

PC12 cells were seeded in a 12-well plate at a density of $1 \times 10^{4}$ cells/well after treated with different concentrations of ropivacaine for $24 \mathrm{~h}$. Then, cells were incubated with Mito-Tracker Red CMXRos (Beyotime, Shanghai, China) according to the instructions. Subsequently, cells were fixed in 4\% PFA, and Hochest33258 was used to label the nuclei. Finally, the fluoresce intensity was observed under a fluorescence microscope and the average optical density was measured by Image J software.

\section{Mitochondrial membrane potential assay}

Mitochondrial membrane potential assay was based on JC-1 staining (5,5',6,6'-tetrachloro-1,1'3,3'tetraethylbenzimidazolcarbocyanine iodide). JC-1 has two forms including monomer and polymer, the emission spectra of which are different. When the mitochondrial membrane potential reduces, the JC- 1 polymer with red fluorescence decreases, and so JC-1 monomer with green fluorescence was in the cytoplasm. Following the instructions of JC-1 assay kit, flow cytometry and fluorescence microscopy were done to measure MMP and the average optical density was determined by ImageJ software.

\section{Mitochondrial separation, extraction of mitochondrial proteins}

The mitochondrial separation was based on differential centrifugation. According to the manufacturer's instructions, the cells were broken down by the Ultrasonic Crusher (Sonic, USA). Then, the cell debris and large organelles were removed by low-speed differential centrifugation $(800 \cdot \mathrm{g})$. Finally, the mitochondria were harvested by high-speed differential centrifugation $(15000 \cdot \mathrm{g})$. The extraction of mitochondrial proteins was done after lysing mitochondria with lysis buffer containing a protease inhibitor and a phosphatase inhibitor. The mitochondrial proteins were used for Western-blot.

\section{Quantitative real-time PCR}


PC12 cells were digested with trypsin and seeded into 6-well plates cultured for 2 days. Then, cells were treated with different concentrations of ropivacaine for $24 \mathrm{~h}$. Total RNA was extracted with Trizol reagent (Invitrogen, USA), and the concentrations were measured by NanoPhotometer (Implen, Germany). A total of $1 \mu \mathrm{g}$ RNA was reversely transcribed into cDNA according to the instructions of RT reagent kit (Promega, USA). SYBR-based real-time PCR experiments were done to detect the mRNA expression of Bax, Bcl-2, Caspase-3, p53, p21, CDK-4, and CyclinE1 using an ABI 7500 platform. The $2^{-\triangle \Delta C T}$ method was used to analyze the data. The primers were used as follows

Bax 5'-CTGAGCTGACCTTGGAGC - 3' (forward), 5'-GACTCCAGCCACA AAGATG-3'(reverse) Bcl-2 5'ATGCCAAGGGGGAAACAC-3' (forward), 5'-CACGGCCGAAAGAGAGA A-3'(reverse) Caspase-3 5'GAAAGCCGAAACTCTTCATCA-3' (forward), 5'-ATAGTAACCG GGTGCGGTAG-3'(reverse), p53 5'GCTGAGTATCTGGACGACAGG-3(forward), 5'-AGCGT GATGATGGTAAGGATG-3' (reverse), p21 5'CTCTGAAGATGTGCCTATGG-3' (forward) 5'-CGAATTTCTCGGACTAATGT-3' (reverse), CDK-4 5'ACCAGGACCTACGGACATAC-3' (forward) 5'-TACAGCCAACACTCCACATA-3' (reverse) CyclinE11 5'-

TGATGATTCAGCG TGCGTGGAC-3' (forward) 5'-CAAGACGGGAAGTGGGGAGG-3' (reverse), GAPDH, 5'GGCTACACTGAGGACCAGGTT-3' (forward) 5'-TGCTGTAGCCATATTCATTGTC-3'(reverse).

\section{Western-Blot}

The PC12 cells were lysed with precold RIPA lysis buffer. After centrifugation (12,000 rpm/min) for 15 min at $4{ }^{\circ} \mathrm{C}$, the protein concentration was measured with a bicinchoninic acid (BCA) protein assay kit (Beyotime, Shanghai, China). A total of $40 \mu \mathrm{g}$ proteins were subjected to protein separation by the sodium dodecyl sulfate-polyacrylamide gel electrophoresis (SDS-PAGE). Proteins were then transferred to a polyvinylidene fluoride (PVDF) membrane. The membranes were incubated with $5 \%$ non-fat skim milk for $1 \mathrm{~h}$. The membranes were incubated with primary antibodies (Bax, 1:1000; Bcl-2, 1:1000; Caspase3, 1:1000; STAT3, 1:1000; p-STAT3 (Ser727), 1:1000; COXIV: 1:1000) at $4^{\circ} \mathrm{C}$ overnight, and then with HRPlinked secondary antibody for $1 \mathrm{~h}$ at room temperature. Specific proteins were detected by enhanced chemiluminescence assay (Bio-rad, USA), and the protein bands were quantified with Image Lab software (Bio-rad, USA).

\section{Detection of cell cycle and apoptosis by flow cytometry}

PC12 cells were seeded in a 6-well plate at a density of $1 \times 10^{6}$ cells/well after treated with different concentrations of ropivacaine for $24 \mathrm{~h}$, cells were collected and centrifuged at $1000 \mathrm{rpm} / \mathrm{min}$ for $5 \mathrm{~min}$ at $4^{\circ} \mathrm{C}$. After washing with PBS, the cell pellet was fixed in $500 \mu \mathrm{L}$ of $70 \%$ cold ethanol for at $2 \mathrm{~h}$. Before flow cytometry, the cells were washed with PBS and incubated with $100 \mu \mathrm{L}$ of RNase. The resulting suspension was incubated at $37^{\circ} \mathrm{C}$ for $30 \mathrm{~min}$. Subsequently, $400 \mu \mathrm{L}$ propidium iodide was added for nuclear staining at $4^{\circ} \mathrm{C}$ in the dark for $30 \mathrm{~min}$. Cell cycles were analyzed using flow cytometry (BD, San Jose, CA). The apoptosis was detected as follows. PC12 cells in each group were digested with trypsin. Then, cells were centrifuged at $1000 \mathrm{rpm} / \mathrm{min}$ for $10 \mathrm{~min}$, and the supernatant was removed. The cells were washed twice with PBS, and centrifuged to remove the supernatant. $500 \mu \mathrm{l}$ of buffer solution was added to the cell suspension. Then, $5 \mu \mathrm{L}$ of Annexin $\mathrm{V}$ and $10 \mu \mathrm{L}$ of propidium iodide were added, 
followed by incubation at room temperature for 30 min in dark, apoptosis was detected by flow cytometry and the apoptosis rate was calculated.

\section{Immunofluorescence staining}

The cell mitochondria were labeled by Mito-Tracker Red CMXRos according to the manufacturer's instructions. Cells were fixed in 4\% PFA and permeabilized with $0.2 \%$ Tween X-100. After being blocked with normal goat serum, cells were incubated with rabbit anti-rat p-STAT3, Cleve-caspase-3 (1:200) overnight at $4^{\circ} \mathrm{C}$, and the second antibody $(1: 1000)$ was incubated for $1 \mathrm{~h}$ at $37^{\circ} \mathrm{C}$ in dark. Hochest 33258 was added followed by incubation for $5 \mathrm{~min}$ to label nuclei. The fluorescence microscope (Olympus, Japan) was used to observe the cells. The fluorescence intensity was analyzed with ImageJ software.

\section{Molecular docking}

Structure-based virtual screening was employed with the molecular docking program Autodock Vina (version 1.1.2). The PyMol (version 2.3) was used to generate the 3D schematic representation of STAT3 protein (PDB ID: 6QHD) which was extracted by using the Research Collaboratory for Structural Bioinformatics Protein Data Bank database. The 2D schematic representation of the interaction between ligand and other amino acid residues was shown by LigPlus (version 2.1). To detect whether ropivacaine competitively binds the STAT3 peptide, the ropivacaine molecular structure (PubChem CID: 175804) was obtained from the PubChem database. The ligand and the receptor pdb were converted to pdbqt file by AutoDock Tools following the autodock vina tutorial, and the grid size in XYZ was set at 96,66,118, which is large enough to cover all potential pockets in the STAT3 monomer.

\section{Animals and treatment}

All procedures performed in studies involving animals were following the ethical standards of the animal ethics committee of the Hubei University of Medicine ((IACUC number: 2018DW003), Eight-week-old Sprague Dawley (SD) rats, weighing 200-300g, were purchased from the Experimental Animal Center of Hubei University of Medicine (Hubei, China). The rats were given access to food and water and housed in an environment with relative humidity of $50-60 \%$ and $12 / 12 \mathrm{~h}$ light/dark cycle for 1 week before surgery. Rats were anesthetized with $10 \%$ chloral hydrate $(300 \mathrm{mg} / \mathrm{kg}$ body weight, Beyotime Biotechnology,

Shanghai, China). The animal was established as previously reported ${ }^{[20]}$. A PE-10 catheter was inserted through the L4 / L5 intervertebral space (depth $2 \mathrm{~cm}$ ) to the L1-L2. The cannulated rats were observed for five days and rats with neurological injury were not included for further experiments. The remaining rats were randomly divided into four groups ( $n=5$ per group), including the control group (saline group), $0.5 \%$ ropivacaine group ( $0.5 \%$ Rop), $1 \%$ ropivacaine group ( $1 \%$ Rop) and $2 \%$ ropivacaine group ( $2 \%$ Rop). All rats have received 8 injections at $1.5 \mathrm{~h}$ intervals for $12 \mathrm{~h}$. After injection, rats were observed for $48 \mathrm{~h}$ and those with movement disorders were removed from this study.

\section{TUNNEL staining and Immunohistochemistry}

The fresh spinal cord tissues were fixed in 4\% PFA. After being embedded with paraffin, the paraffin blocks were cut into $3 \mathrm{~mm}$ slices. Then, they were detected with TUNNEL staining according to the 
manufacturer's instruction to detect the apoptosis in the spinal cord. The protein expression of p-STAT3 (Ser727) in the spinal cord was detected by Immunohistochemistry.

\section{Statistical analysis}

Statistical analysis was performed using SPSS version 22.0 software. The quantitative data were expressed as mean \pm standard deviation (SD). The comparisons between the two groups were done with Student's t-test. The comparison among groups was performed by one-way ANOVA. A value of $P<0.05$ was considered statistically different.

\section{Results}

\section{Ropivacaine decreased viability and reduced the protrusion amounts in PC12 cells}

To determine the effect of ropivacaine on PC12 cell viability, CCK-8 assay was done after the treatment with different ropivacaine concentrations $(0.5 \mathrm{mM}, 1 \mathrm{mM}, 2 \mathrm{mM}, 4 \mathrm{mM}, 8 \mathrm{mM})$ for $24 \mathrm{~h}$. As shown in Fig. 1, compared with the control group, ropivacaine suppressed PC12 cell viability in a dose-dependent manner, and the $\mathrm{IC}_{50}$ was $3.24 \mathrm{mM}$. Under the optical microscope, the morphology of cells in the ropivacaine group has been changed, which was round and whose protrusion reduced. Those results indicate that ropivacaine exerted significant toxic effects on PC12 cells by inhibiting cell viability.

\section{Ropivacaine induced PC12 cell cycle arrest in the G0/G1 phase}

To explore the effect of ropivacaine on the cell death pathway, the cell cycle was detected by flow cytometry before and after the treatment with ropivacaine. As shown in Fig. 2A, 2B, after exposure to different concentrations of ropivacaine for $24 \mathrm{~h}$, the proportion of cells in the G0/G1 phase was significantly increased, and that in the G2/M phase was decreased compared to the control group, but ropivacaine had no influence on the $S$ phase. In addition, ropivacaine upregulated the mRNA and protein expression of p53 and p21, and inhibited Cyclin E1 and CDK4 levels, which was further to verify that ropivacaine induced $\mathrm{G} 1$ phase arrest in PC12 cells (Fig. 2C-2E).

\section{Ropivacaine induced the apoptosis of PC12 cells and the spinal cord}

In addition to detecting the cell cycle, the levels of apoptosis rate in PC12 cells were also detected by flow cytometry, As shown in Fig. 3D, compared with the control group, ropivacaine increased the levels of apoptosis of PC12 cells in a dose-dependent manner, and it also up-regulated the mRNA and protein expression of pro-apoptotic genes Bax and cleve-caspase-3, and down-regulated anti-apoptotic gene Bcl2 levels. To further illustrate the neurotoxicity of ropivacaine on the spinal cord, a rat model was established with a subarachnoid catheter. After the treatment with different concentrations of ropivacaine, the levels of apoptosis in the spinal cord were detected by TUNNEL staining. The results showed that the apoptosis rate in the spinal cord in the ropivacaine group was increased significantly to the control group (Fig. 3E, 3F).

\section{Ropivacaine induced oxidative stress in PC12 cells}


To further access the effects of ropivacaine on the levels of oxidative stress in PC12 cells, the SOD and LDH activities, MDA content, and intercellular ROS levels were detected. As shown in Fig. 4A, compared with the control group, ropivacaine increased the intercellular ROS levels in a dose-dependent manner. In addition, ropivacaine promoted the oxidative stress of PC12 cells via enhancing the LDH activity and MDA content, and decreasing the SOD activity ((Fig. 4B-4E).

\section{Ropivacaine induced mitochondrial dysfunction in PC12 cells}

To assess the effect of ropivacaine on mitochondria in PC12 cells, the fluorescent dye JC-1 was to detect the $\Delta \psi \mathrm{m}$ under the flow cytometry and fluorescence microscopy. As shown in Fig. 5A, compared with the control group, the proportion of cells in the red fluorescence channel (PE channel) gradually decreased in ropivacaine group, which indicated that ropivacaine decreased the $\triangle \Psi \mathrm{m}$ of PC12 cells. The results from fluorescence microscopy were consistent with the flow cytometry. A significantly lower red/green fluorescence rate was observed in the ropivacaine group compared with the control group (Fig. 5B, 5C). Suggesting that ropivacaine induced the depolarization and permeabilization of mitochondria of PC12 cells. Moreover, to further illustrate the effects of ropivacaine on mitochondrial amount, the mitochondria was also detected by Mito-tracker Red CMXRos following ropivacaine treatment and depicted the normal mitochondrial staining. As shown in Fig. 5D,5E, compared with the control group, ropivacaine treatment significantly reduced the mitochondrial numbers with normal function in PC12 cells. Finally, the mtDNA/nDNA ratio kit was also determined to assess the mitochondrial numbers, the results from Fig. 5F showed that ropivacaine reduced the mtDNA/nDNA ratio in a dose-dependent manner in PC12 cells. The above results demonstrated that ropivacaine induced the mitochondrial dysfunction of PC12 cells.

\section{Ropivacaine inhibited STAT3 Ser727 phosphorylation and mitoSTAT3 translocation}

To illustrate the effect of ropivacaine on the STAT3 signaling pathway, the expression of STAT3 phosphorylation and upstream transduction protein were evaluated using Western-blot, and the distribution of phosphorylated STAT3 in subcellular compartments was also detected. As shown in Fig. 6A and Fig. 6D, ropivacaine inhibited the p-STAT3 (Ser 727) expression and down-regulated IL-6 levels in PC12 cells. Moreover, compared with the control group, ropivacaine decreased p-STAT3 (ser727) expression in the mitochondrial fraction but increased it in the cytoplasmic fraction (Fig. 6B, 6E). Suggesting that ropivacaine inhibited the mitoSTAT3 translocation. The result of immunofluorescence was consistent with Western-blot, ropivacaine reduced the co-localization between p-STAT3 (ser727) with mitochondria in a dose-dependent manner (Fig. 6C). In addition, ropivacaine inhibited the p-STAT3 (Ser727) expression in the spinal cord tissues in a dose-dependent manner (Fig. 6G, 6H). Finally, the autodock-Vina was to examine the interaction between STAT3 and ropivacaine. As shown in Fig. 6F, the pocket was composed of Asn257, Ala377, Glu324, Ile258, Gln326, Pro333, Pro336, Asp 334, Ala250, Cys251 and Pro256. They interacted through hydrophobic bonds to affect the activation of STAT3.

\section{Reduced mitoSTAT3 translocation enhanced ropivacaine-induced apoptosis}

To further illustrate that mitoSTAT3 translocation was involved in ropivacaine-induced apoptosis, PC12 cells were pretreated with the inhibitor (S31201) and the activator (IL-6) of the mitoSTAT3 translocation, and the co-localization between p-STAT3 (ser727) with mitochondria was also detected, as shown in 
Fig. 7A, S31201 inhibited the phosphorylation of STAT3 at Ser727 in PC12 cells, and it also decreased the co-localization of p-STAT3 (ser727) and mitochondria, so that enhanced the inhibition of ropivacaine on mitoSTAT3 translocation. On the contrary, IL-6 was used to activate STAT3 signaling pathway, it enhanced STAT3 Ser727 phosphorylation and the co-localization of p-STAT3 (ser727) and mitochondria, so that reversed the effect of ropivacaine on mitoSTAT3 translocation. Comparing the effects of the mitoSTAT3 translocation inhibited or activated on ropivacaine-induced apoptosis, the immunofluorescence staining of Cle-Caspase-3 was detected, as shown in Fig. 7B, compared with the ropivacaine group, the green fluorescence intensity represented Cle-Caspase-3 protein expression was significantly increased in the S31201 + ropivacaine group, suggesting that S31201 enhanced ropivacaineinduced apoptosis. On the contrary, IL-6 pretreatment decreased the expression of Cle-Caspase-3 in PC12 cells, so that reversed ropivacaine-induced apoptosis.

\section{Discussion}

Ropivacaine, as a representative of the long-acting local anesthetics (LAs), is available for spinal anesthesia, and its duration is $1.6-6 \mathrm{~h}^{[21]}$. However, some clinical studies have shown that ropivacaine may induce severe neurotoxic side effects after intravenous injection in recent years. Some experiments have also demonstrated that ropivacaine induces neurotoxicity after long-term exposure to ropivacaine at a high concentration ${ }^{[22-24]}$. Since Yamashita et al. first reported the neurotoxicity of ropivacaine [25], many studies have been conducted to explore and compare the neurotoxicity of LAs commonly used in clinical practice, including lidocaine, and bupivacaine ${ }^{[26,27]}$. Although it had been reported that ropivacaine has the least neurotoxicity among the LAs tested, increasing attention has been paid to the safety of ropivacaine ${ }^{[25]}$. Currently, the clinical dosage of ropivacaine is still controversial. It has been reported that injection of high-dose ropivacaine into the epidural space or subarachnoid space can shorten the onset time and increase the motor block effect ${ }^{[28]}$, while other studies have shown the increased risk of neurotoxicity after exposure to high-dose ropivacaine ${ }^{[29]}$. Hence, it is important to investigate the mechanism underlying the ropivacaine-induced neurotoxicity, which is helpful for the development of preventive measures and will make spinal anesthesia safer.

The mechanism of ropivacaine-induced neurotoxicity is unclear, and some studies reported that it is related to cell apoptosis. Our previous study also revealed that ropivacaine increased apoptosis via upregulating Fas and Fasl expression in PC12 cells ${ }^{[8]}$, which were also confirmed by Wang et al. ${ }^{[30]}$. Moreover, apoptosis may execute in two pathways: the death receptor pathway and the mitochondrial pathway ${ }^{[8]}$. The death receptor pathway is represented by the up-regulation of Fas and Fasl expression, and the mitochondrial pathway is characterized by mitochondrial dysfunction. In addition to inducing neurotoxicity through the death receptor pathway, ropivacaine has also been proved to cause neuronal damage by impairing mitochondrial function. Niu et al. reported ropivacaine affected the mitochondrial biogenesis of neuronal cells by reducing the mitochondrial mass and impairing the mitochondrial respiratory rate via suppressing PCG-1a ${ }^{[31]}$. In the present study, our results confirmed that apoptosis played an important role in ropivacaine-induced neurotoxicity. The results from CCK-8 assay showed that 
ropivacaine decreased cell viability in a dose-dependent manner. In addition, ropivacaine reduced the protrusion of PC12 cells and increased the death cell amount, and it also induced PC12 cells in the G0/G1 phase arrest and apoptosis. Except for the above indicators, the mRNA and protein expression of genes related to cell cycle and apoptosis were also detected. p21 and p53 are those genes that block the cell cycle progression ${ }^{[32]}$, CyclinE1 and CDK4 are the key genes for the maintenance of normal cell cycle progression ${ }^{[33-35]}$, ropivacaine treatment blocked the cell cycle progression at G0/G1 phase via the upregulation of p53 and p21 levels, and the down-regulation of CyclinE1 and CDK4 expression. Bax and Caspase 3 are the pro-apoptotic proteins in the mitochondrial apoptosis pathway, and Bcl-2 is an antiapoptotic protein. Our results also demonstrated ropivacaine treatment increased the expression of Bax and Caspase 3 in PC12 cells and inhibited Bcl-2 levels, suggesting that the mitochondrial apoptosis pathway is involved in the ropivacaine-induced neurotoxicity.

To further clarify the effects of ropivacaine on cellular oxidative stress and mitochondrial function, the reactive oxygen species (ROS) generation was assessed in cells before and after the treatment with ropivacaine. The results showed that ropivacaine increased the levels of ROS. Similarly, the contents of MDA and LDH also increased significantly by ropivacaine treatment, while the level of SOD decreased after ropivacaine treatment. Suggesting that ropivacaine induced cellular oxidative stress in PC12 cells. Those results were consistent with Chen et al ${ }^{[5]}$. Moreover, we also confirmed that ropivacaine induced mitochondrial dysfunction, which was mainly manifested in the decrease of $\psi \Delta \mathrm{m}$ and $\mathrm{mtDNA} / \mathrm{nDNA}$ ratio. It was also confirmed by other studies ${ }^{[36,31]}$.

On the basis of above results, the effect of ropivacaine on the STAT3 signaling pathway was to illustrate. STAT3 was first described as a transcriptional enhancer of acute-phase genes activated by IL-6, which mediated extracellular signals such as cytokines and growth factors through interacting with polypeptide receptors on the cell surface ${ }^{[37]}$. The canonical STAT3 signaling becomes transcriptionally activated primarily by tyrosine phosphorylation (Tyr705). Activated STAT3 translocates to the nucleus and binds to sequence-specific DNA elements with consequent transcription of target genes ${ }^{[38]}$. The canonical STAT3 pathway has been demonstrated to promote tumor development ${ }^{[13]}$. The non-canonical STAT3 signaling is represented by serine phosphorylation (Ser727), and the function of STAT3 Ser727 phosphorylation is to rescue the impaired mitochondrial function after exposing to the damage $\mathrm{e}^{[14]}$. When apoptosis is induced via the mitochondrial pathway, the non-canonical STAT3 signaling is activated to further protect cells against apoptosis. Except for the non-canonical pathway, STAT3 has been shown to regulate the mitochondrial function by transporting it to the mitochondria, which is also dependent on serine phosphorylation (Ser727) ${ }^{[17,15]}$. Our results found that ropivacaine inhibited the STAT3 Ser727 phosphorylation and the expression of IL-6 protein. We also demonstrated that ropivacaine inhibited the mitoSTAT3 translocation in a dose-dependent manner. Although the anti-apoptotic effects of mitoSTAT3 have been reported by many studies. Li et al. reported that the STAT3 activation is key for ischemic postconditioning, and the increase of mitoSTAT3 can protect the cardiomyocytes against mitochondrial dysfunction ${ }^{[39]}$. Zhang et al. reported Serine 727 (Ser727) phosphorylation of STAT3 plays a role in the regulation of mitochondrial respiration ${ }^{[40]}$. In addition, mitoSTAT3 translocation can improve oxidative 
phosphorylation and inhibit ROS generation during reperfusion. Moreover, our results also demonstrated that ropivacaine could bind to the hydrophobic domain of STAT3's proline site and other sites. And the results showed that ropivacaine increased the apoptotic levels and decreased the protein expression of $p$ STAT3 (Ser727) in the spinal cord tissues in vivo experiment. Finally, we also demonstrated that the mitoSTAT3 translocation inhibitor S31201 could enhance ropivacaine-induced apoptosis, but the activator IL-6 reversed this effect.

\section{Conclusion}

In conclusion, ropivacaine induced neurotoxicity via decreasing cell viability and the protrusions of PC12 cells. Moreover, ropivacaine blocked the cell cycle progression at G0/G1 phase and promoted cell apoptosis through the mitochondrial apoptosis pathway. And it also induced oxidative stress and mitochondrial dysfunction in PC12 cells. These effects may be related to the inhibition of STAT3 activation and mitoSTAT3 translocation. These findings suggest STAT3 may serve as a target in the prevention a treatment of ropivacaine induced neurotoxicity

\section{Declarations}

\section{Funding}

The Innovative Research Program for Graduates of Hubei University of medicine (NO. YC2020029), Hubei Natural Science Foundation (No. 2019CFB105)

\section{Competing Interests}

The authors declare no conflicts of interest regarding this study and publication.

\section{Authors' Contributions}

LHY and DXD designed the studies, ZL and ZZ undertook the cell experiments and the construction of animal experiments, ZL and ZFY undertook the molecular biology testing, ZL, CHX, WY undertook the molecular docking. ZL, ZFY, ZZ analyzed data and wrote the draft of manuscript.

\section{Ethical Approval And Consent To Participate}

All procedures performed in studies involving animals were in accordance with the ethical standards of the institution or practice at the Animal Ethics Committee of the Hubei University of Medicine. (permit number: 2018DW003)

\section{Availability of data and materials}

Data that support the study findings are available from the corresponding author upon reasonable request. 
Acknowledgements

Not applicable

\section{Consnt For Publication}

The authors declare the consnt for publication

\section{References}

[1] Martin-Flores M. Epidural and Spinal Anesthesia[J].The Veterinary clinics of North America. Small animal practice. 2019, 49: 1095-1108.

[2] Cai XY, Xia Y, Yang SH, et al. Ropivacaine- and bupivacaine-induced death of rabbit annulus fibrosus cells in vitro: involvement of the mitochondrial apoptotic pathway[J].Osteoarthritis and cartilage. 2015, 23: $1763-1775$.

[3] CM C, GR T, LG M, et al. Local neurotoxicity and myotoxicity evaluation of cyclodextrin complexes of bupivacaine and ropivacaine[J].Anesthesia and analgesia. 2012, 115: 1234-1241.

[4] Aps J, Badr N. Narrative review: the evidence for neurotoxicity of dental local anesthetics[J].Journal of dental anesthesia and pain medicine. 2020, 20: 63-72.

[5] Chen Y, Yan L, Zhang Y, et al. The role of DRP1 in ropivacaine-induced mitochondrial dysfunction and neurotoxicity[J].Artificial cells, nanomedicine, and biotechnology. 2019, 47: 1788-1796.

[6] Wang S, Lin Q, Wang Z, et al. Ropivacaine induces neurotoxicity by activating MAPK/p38 signal to upregulate Fas expression in neurogliocyte[J].Neurosci Lett. 2019, 706: 7-11.

[7] Wen X, Li Y, Liu X, et al. Roles of CaMKIIß in the neurotoxicity induced by ropivacaine hydrochloride in dorsal root ganglion[J].Artificial cells, nanomedicine, and biotechnology. 2019, 47: 2948-2956.

[8] Zeng Y, Wang R, Bian Y, et al. Catalpol Attenuates IL-1 $\beta$ Induced Matrix Catabolism, Apoptosis and Inflammation in Rat Chondrocytes and Inhibits Cartilage Degeneration[J].Medical science monitor : international medical journal of experimental and clinical research. 2019, 25: 6649-6659.

[9] Sgrignani J, Garofalo M, Matkovic M, et al. Structural Biology of STAT3 and Its Implications for Anticancer Therapies Development[J].Int J Mol Sci. 2018, 19.

[10] Guanizo AC, Fernando CD, Garama DJ, et al. STAT3: a multifaceted oncoprotein[J].Growth factors (Chur, Switzerland). 2018, 36: 1-14.

[11] Hillmer EJ, Zhang H, Li HS, et al. STAT3 signaling in immunity[J].Cytokine \& growth factor reviews. 2016, 31: 1-15. 
[12] Vogel TP, Milner JD, Cooper MA. The Ying and Yang of STAT3 in Human Disease[J].Journal of clinical immunology. 2015, 35: 615-623.

[13] Fathi N, Rashidi G, Khodadadi A, et al. STAT3 and apoptosis challenges in cancer[J].International journal of biological macromolecules. 2018, 117: 993-1001.

[14] Srivastava J, DiGiovanni J. Non-canonical Stat3 signaling in cancer[J].Molecular carcinogenesis. 2016, 55: 1889-1898.

[15] Yang R, Rincon M. Mitochondrial Stat3, the Need for Design Thinking[J].International journal of biological sciences. 2016, 12: 532-544.

[16] Heusch G, Musiolik J, Gedik N, et al. Mitochondrial STAT3 activation and cardioprotection by ischemic postconditioning in pigs with regional myocardial ischemia/reperfusion[J].Circulation research. 2011, 109: 1302-1308.

[17] Szczepanek K, Chen Q, Derecka M, et al. Mitochondrial-targeted Signal transducer and activator of transcription 3 (STAT3) protects against ischemia-induced changes in the electron transport chain and the generation of reactive oxygen species[J].The Journal of biological chemistry. 2011, 286: 2961029620.

[18] Flanagan SE, Haapaniemi E, Russell MA, et al. Activating germline mutations in STAT3 cause earlyonset multi-organ autoimmune disease[J].Nature genetics. 2014, 46: 812-814.

[19] Steward-Tharp SM, Laurence A, Kanno Y, et al. A mouse model of HIES reveals pro- and antiinflammatory functions of STAT3[J].Blood. 2014, 123: 2978-2987.

[20] Y H, L W, J G, et al. A modified procedure for lumbar intrathecal catheterization in rats[J].Neurological research. 2016, 38: 725-732.

[21] Z S, H L, Q G, et al. In vivo and in vitro evidence of the neurotoxic effects of ropivacaine: the role of the Akt signaling pathway[J].Molecular medicine reports. 2012, 6: 1455-1459.

[22] A B, S F, G L, et al. Transient neurologic symptoms (TNS) after intrathecal injection of ropivacaine through a dural tap during an attempted epidural for labour pain relief[J].Anaesthesia, critical care \& pain medicine. 2017, 36: 325-326.

[23] S C, S S. The role of local anaesthetic techniques in ERAS protocols for thoracic surgery[J].Journal of thoracic disease. 2018, 10: 1998-2004.

[24] W Z, BM G. The toxicity of local anesthetics: the place of ropivacaine and levobupivacaine[J].Current opinion in anaesthesiology. 2008, 21: 645-650. 
[25] Yamashita A, Matsumoto M, Matsumoto S, et al. A comparison of the neurotoxic effects on the spinal cord of tetracaine, lidocaine, bupivacaine, and ropivacaine administered intrathecally in rabbits[J].Anesthesia and analgesia. 2003, 97: 512-519, table of contents.

[26] Byram SC, Bialek SE, Husak VA, et al. Distinct neurotoxic effects of select local anesthetics on facial nerve injury and recovery[J].Restorative neurology and neuroscience. 2020, 38: 173-183.

[27] Lirk P, Haller I, Colvin HP, et al. In vitro, inhibition of mitogen-activated protein kinase pathways protects against bupivacaine- and ropivacaine-induced neurotoxicity[J].Anesthesia and analgesia. 2008, 106: $1456-1464$, table of contents.

[28] Guryay D, Karaege G, Katircioglu K, et al. The effects of an epidural infusion of ropivacaine versus saline on sensory block after spinal anesthesia[J].Regional anesthesia and pain medicine. 2008, 33: 217221.

[29] Takenami T, Yagishita S, Asato F, et al. Intrathecal lidocaine causes posterior root axonal degeneration near entry into the spinal cord in rats[J].Regional anesthesia and pain medicine. 2002, 27: 58-67.

[30] S W, Q L, Z W, et al. Ropivacaine induces neurotoxicity by activating MAPK/p38 signal to upregulate Fas expression in neurogliocyte[J].Neuroscience letters. 2019, 706: 7-11.

[31] Z N, J T, Y R, et al. Ropivacaine impairs mitochondrial biogenesis by reducing PGC-1a[J].Biochemical and biophysical research communications. 2018, 504: 513-518.

[32] El-Deiry WS. p21(WAF1) Mediates Cell-Cycle Inhibition, Relevant to Cancer Suppression and Therapy[J].Cancer research. 2016, 76: 5189-5191.

[33] Lim S, Kaldis P. Cdks, cyclins and CKIs: roles beyond cell cycle regulation[J].Development (Cambridge, England). 2013, 140: 3079-3093.

[34] Schade AE, Oser MG, Nicholson HE, et al. Cyclin D-CDK4 relieves cooperative repression of proliferation and cell cycle gene expression by DREAM and RB[J].Oncogene. 2019, 38: 4962-4976.

[35] Xu W, McArthur G. Cell Cycle Regulation and Melanoma[J].Current oncology reports. 2016, 18: 34.

[36] Y C, L Y, Y Z, et al. The role of DRP1 in ropivacaine-induced mitochondrial dysfunction and neurotoxicity[J].Artificial cells, nanomedicine, and biotechnology. 2019, 47: 1788-1796.

[37] You L, Wang Z, Li H, et al. The role of STAT3 in autophagy[J].Autophagy. 2015, 11: 729-739.

[38] Cui P, Wei F, Hou J, et al. STAT3 inhibition induced temozolomide-resistant glioblastoma apoptosis via triggering mitochondrial STAT3 translocation and respiratory chain dysfunction[J].Cellular signalling. 2020, 71: 109598. 
[39] Li H, Yao W, Liu Z, et al. Hyperglycemia Abrogates Ischemic Postconditioning Cardioprotection by Impairing AdipoR1/Caveolin-3/STAT3 Signaling in Diabetic Rats[J].Diabetes. 2016, 65: 942-955.

[40] Zhang G, Sheng M, Wang J, et al. Zinc improves mitochondrial respiratory function and prevents mitochondrial ROS generation at reperfusion by phosphorylating STAT3 at Ser(727)[J].Journal of molecular and cellular cardiology. 2018, 118: 169-182.

\section{Figures}
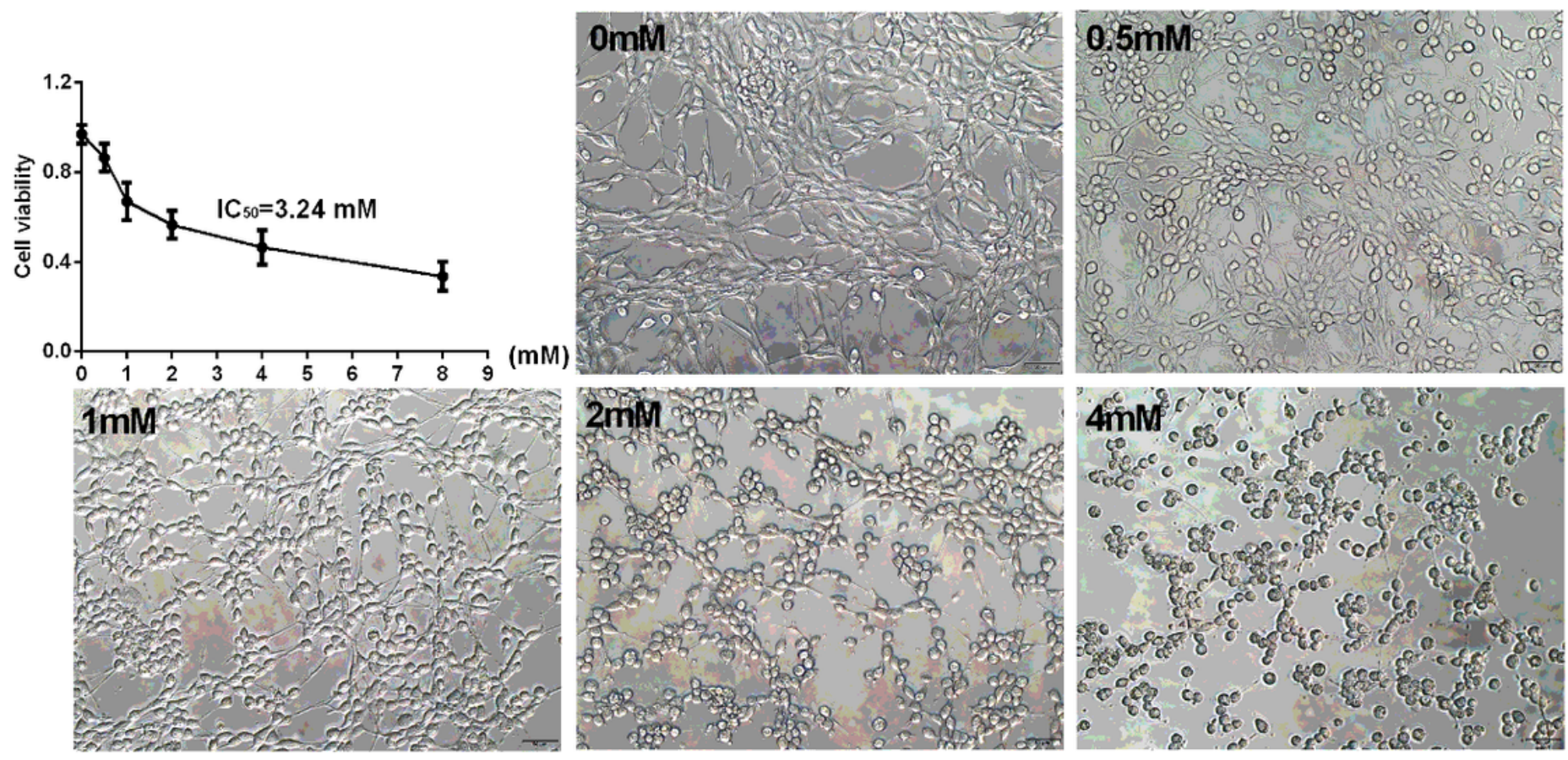

\section{Figure 1}

Ropivacaine treatment decreased viability and reduced the protrusions of PC12 cells. (A) PC12 cells were treated with various concentrations of ropivacaine $(0.5 \mathrm{mM}, 1 \mathrm{mM}, 2 \mathrm{mM}, 4 \mathrm{mM}, 8 \mathrm{mM})$ for $24 \mathrm{~h}$, the cell viability was detected by CCK-8. (B) PC12 cells were treated as above, the cell morphology was observed by microscope $(x 200)$. The results are presented as the mean $\pm S D(n=3)$, where $* p<0.05,{ }^{\star *} p<0.01$ compared with the control. 
A

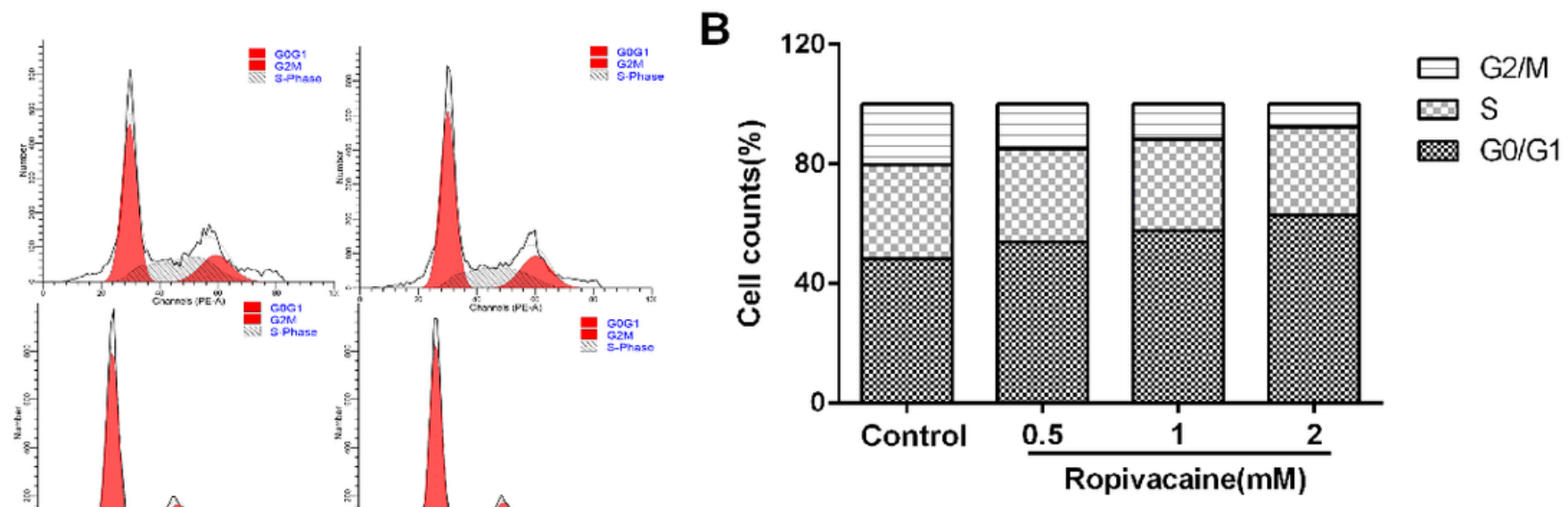

C
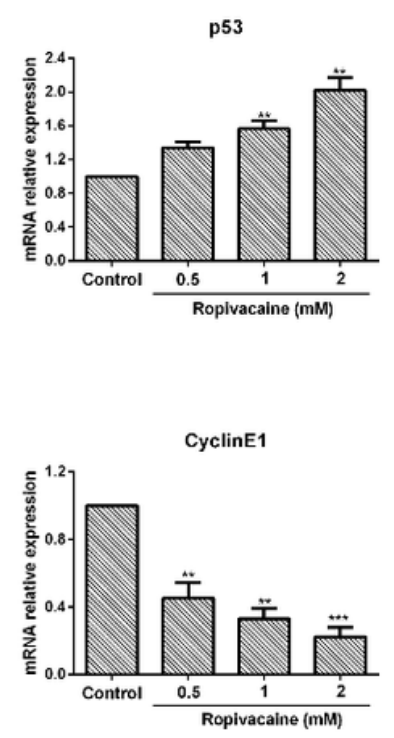

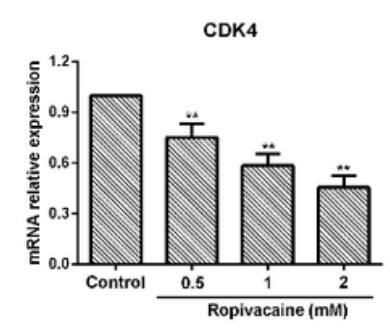

D

p21

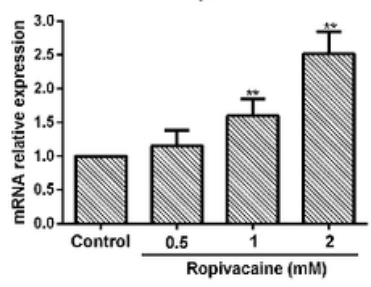

$E$
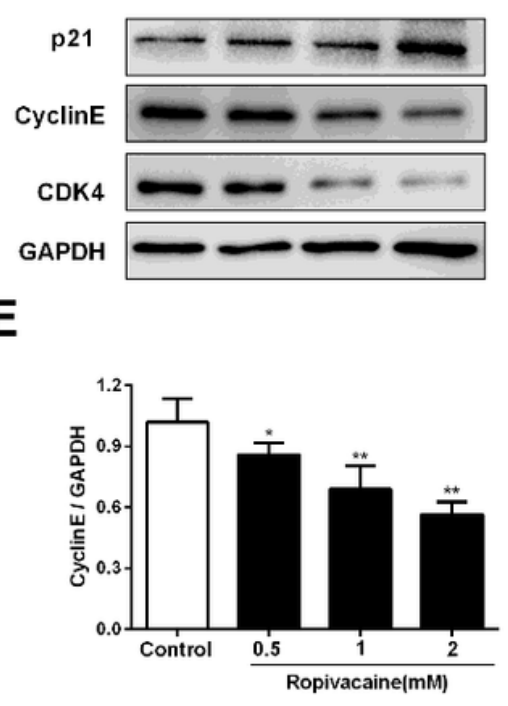
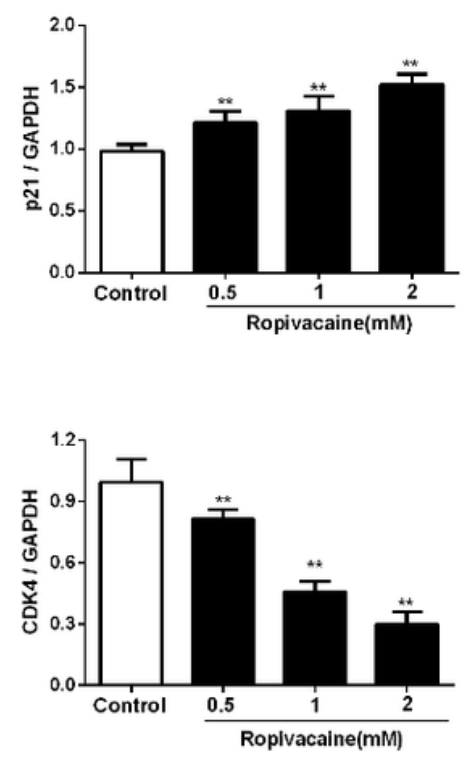

Figure 2

Ropivacaine treatment blocked the cell cycle progression at G0/G1 phase via the up-regulation of p53 and p21 levels, and the down-regulation of CyclinE1 and CDK4 expression. (A) PC12 cells were treated with different concentrations of ropivacaine, the cell cycle was detected by flow cytometry. (B) the quantification of the cell cycle included G0/G1 phase, S phase and G2/M phase. (C) The mRNA expression of p53, p21, CDK4, CyclinE1 were detected by qRT-PCR. (D) The protein expression of p21, CDK4, CyclinE1 were detected by Western-blot. (E) Quantification of the gray values in graph $\mathrm{D}$. The results are presented as the mean $\pm S D(n=3)$, where ${ }^{*} p<0.05$, ${ }^{\star *} p<0.01$ compared with the control. 
A

Bax
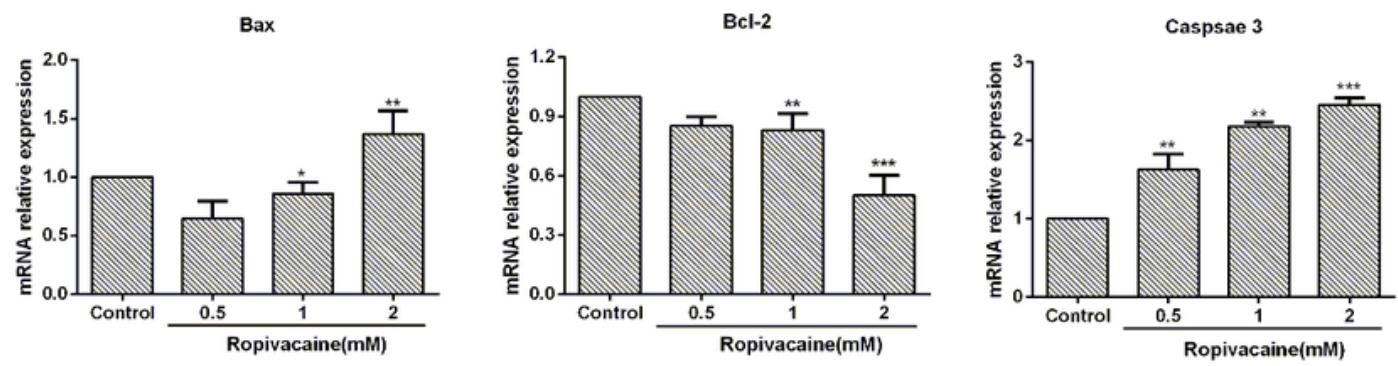

B
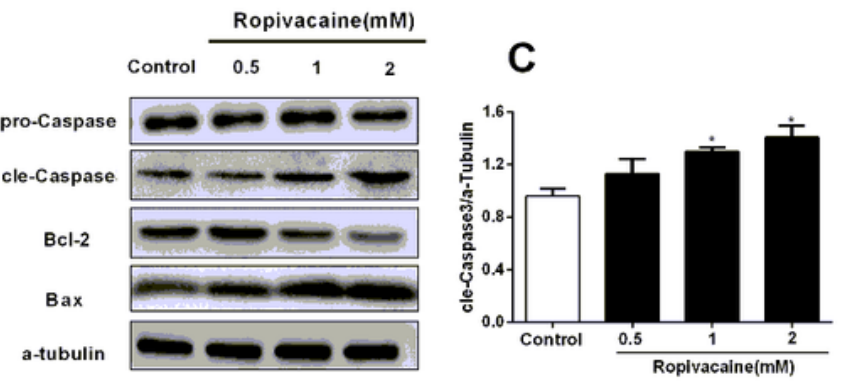

D
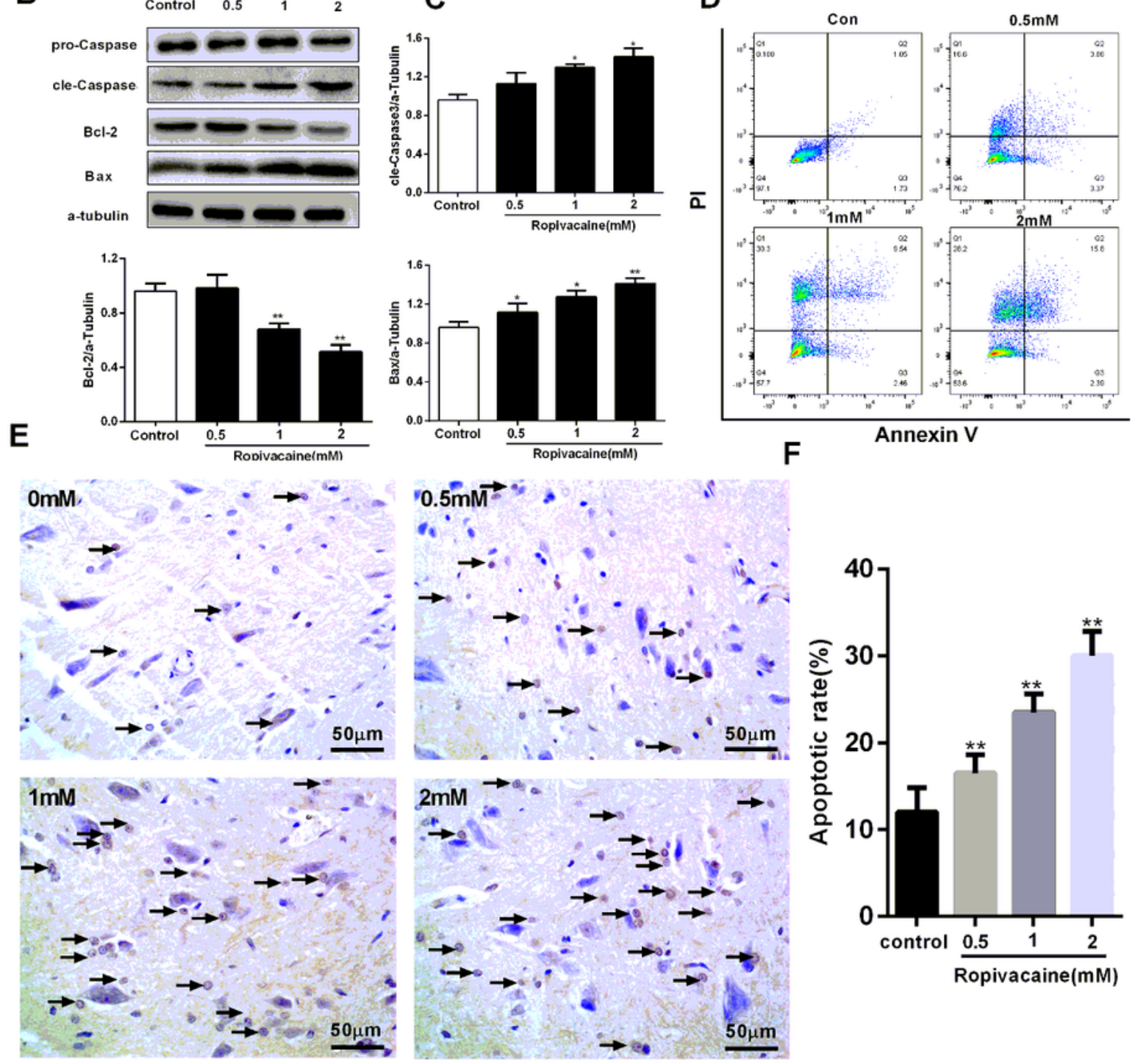

Figure 3

Ropivacaine induced apoptosis of PC12 cells and the spinal cord tissues (A) The mRNA expression of Bax, Bcl-2 and caspase 3 in PC12 cells were detected by qRT-PCR. (B) The protein expression of Bax, Bcl2, Caspase 3 and Cleaved-caspase-3 in PC12 cells was detected by Western-blot. (C) Quantification of the gray values in graph B. (D) The levels of apoptosis were detected by flow cytometry. (E) Rats have received the subarachnoid catheterization, and injected with different concentrations of ropivacaine, the 
apoptosis of the spinal cord was detected by TUNNEL staining. Arrows refer to apoptotic cells. (F) The ratio of the numbers of apoptotic cells to total cells. The results are presented as the mean $\pm S D(n=3)$, where $* p<0.05, * * p<0.01, * \star \star p<0.001$ compared with the control.
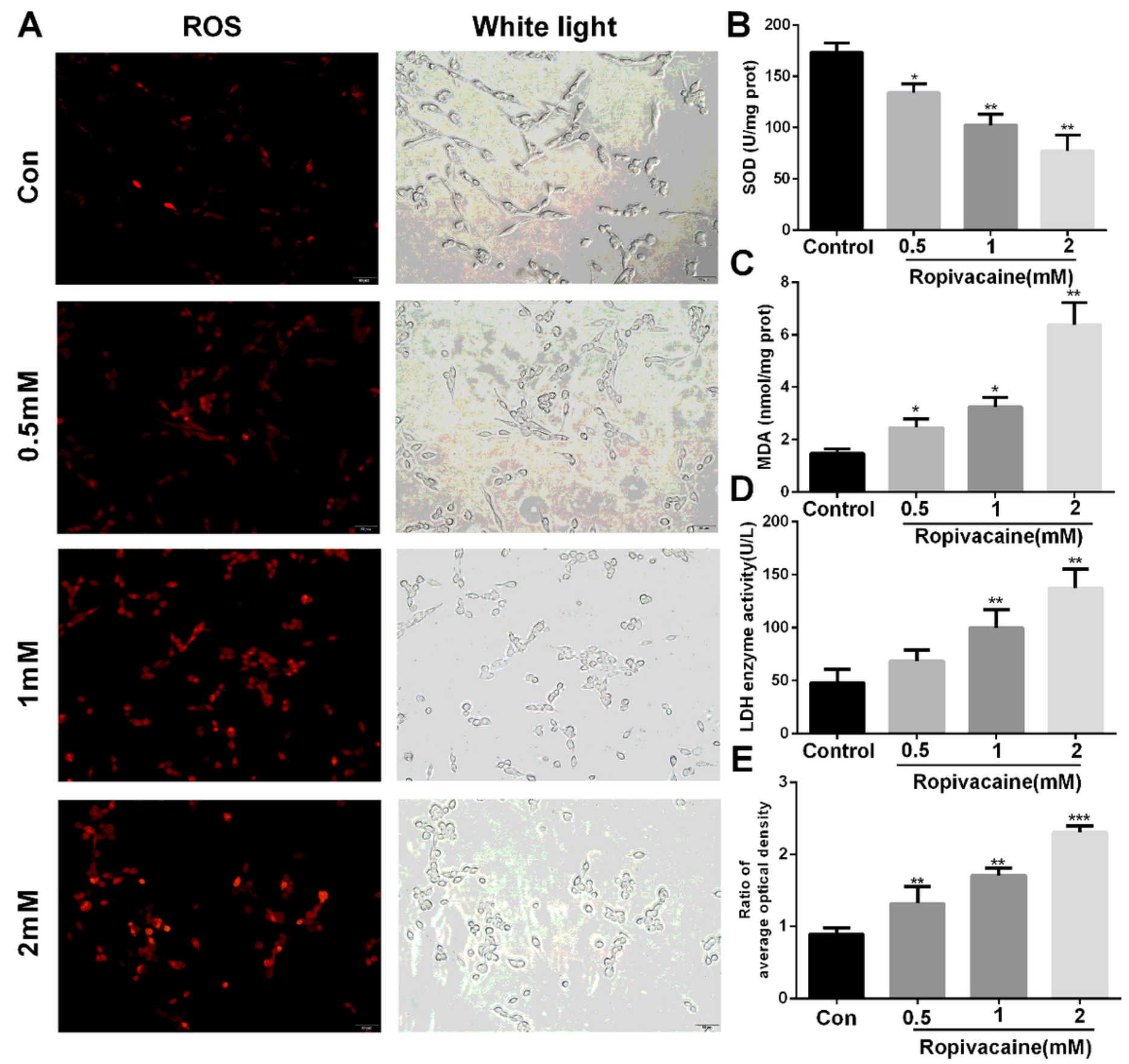

\section{Figure 4}

Ropivacaine induced oxidative stress in PC12 cells. (A) PC12 cells were treated with different concentrations of ropivacaine, Intracellular ROS was determined by DHC assay. (B) (C) (D) SOD, MDA and LDH were detected by the assay kits. (E) The ratio of the average optical density in graph A. The results are presented as the mean $\pm S D(n=3)$, where $* p<0.05$, ${ }^{\star *} p<0.01$, ${ }^{\star \star \star} p<0.001$ compared with the control. (F) 


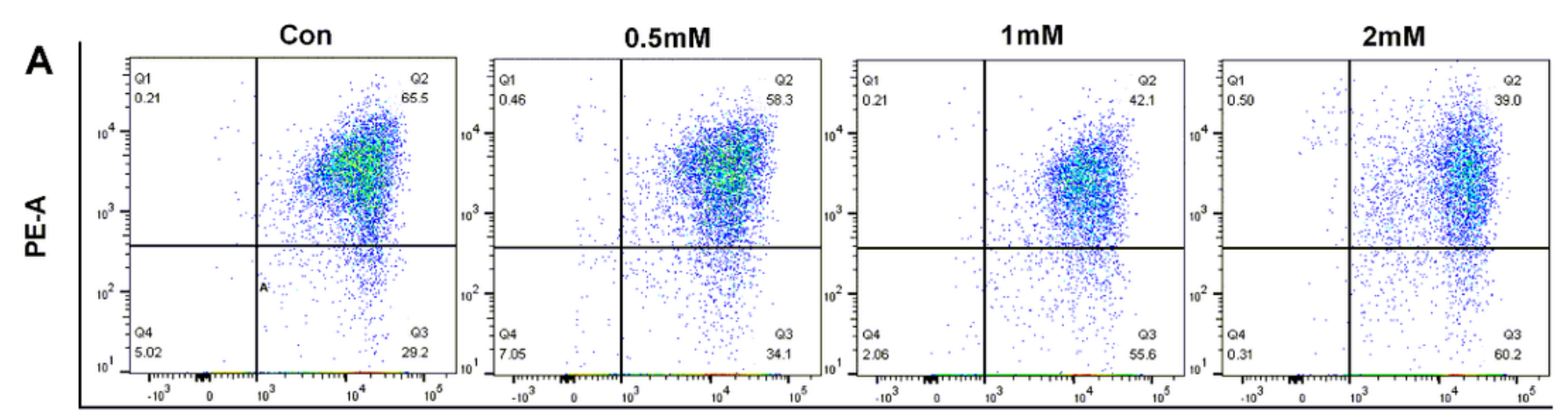

B

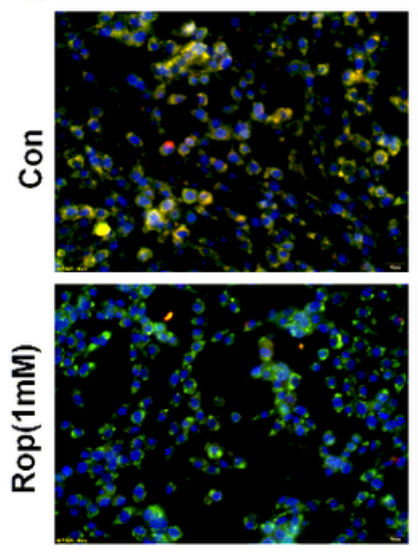

Merge
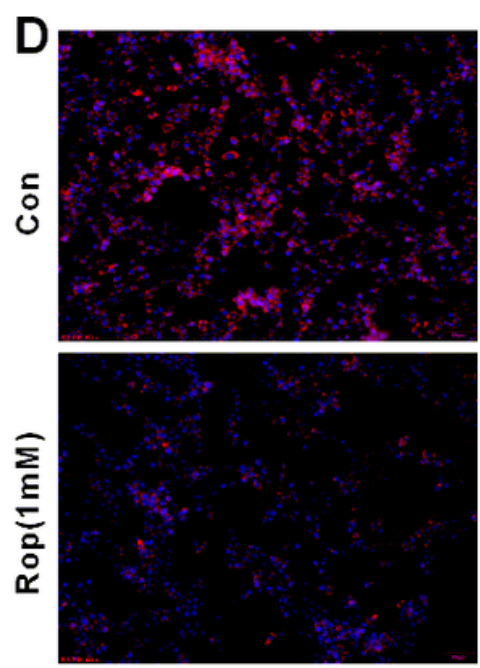

Merge

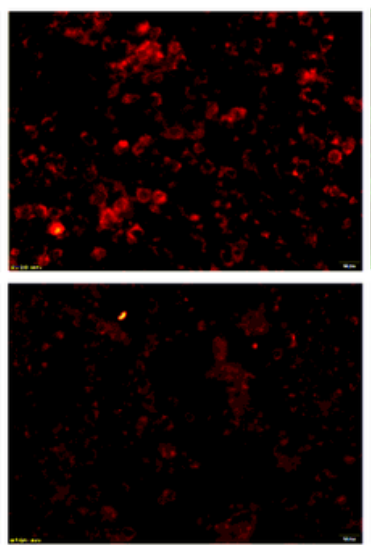

JC-1 polmer
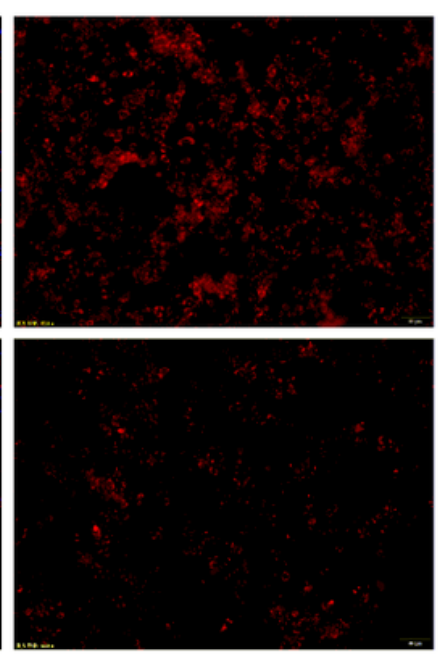

Mito-traker

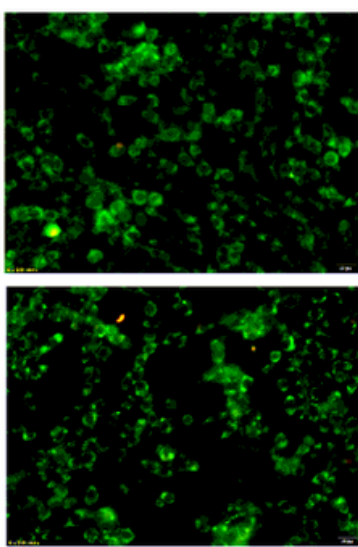

JC-1 monomer

C

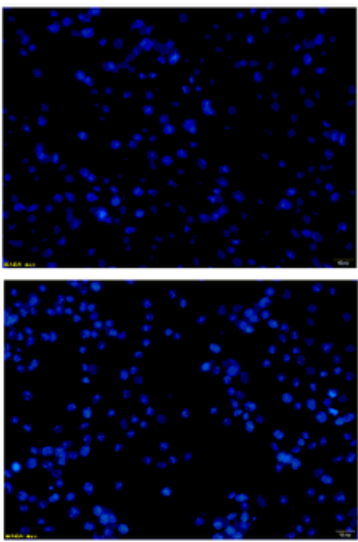

Hochest33258
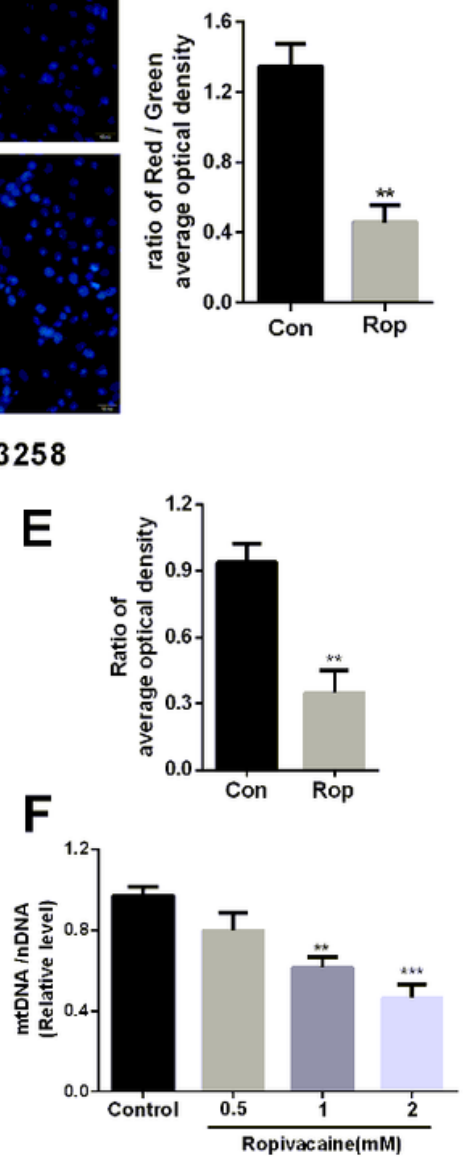

Hochest 33258

\section{Figure 5}

Ropivacaine induced PC12 cells mitochondrial dysfunction. (A) (B) PC12 were treated with different concentrations of ropivacaine, mitochondrial function was detected by a JC-1 assay kit and quantified by the flow cytometry, and observed in a fluorescence microscope. (C) The ratio of Red / Green average optical density in graph B. (D) PC12 cells mitochondrial was labeled by the Mito-Tracker Red CMXRos and observed in a fluorescence microscope. (E) The ratio of mtDNA to nDNA (mtDNA/nDNA) was 
measured by qRT-RCR. The results are presented as the mean $\pm S D(n=3)$, where ** $p<0.01, * \star \star p<0$ .001 compared with the control.
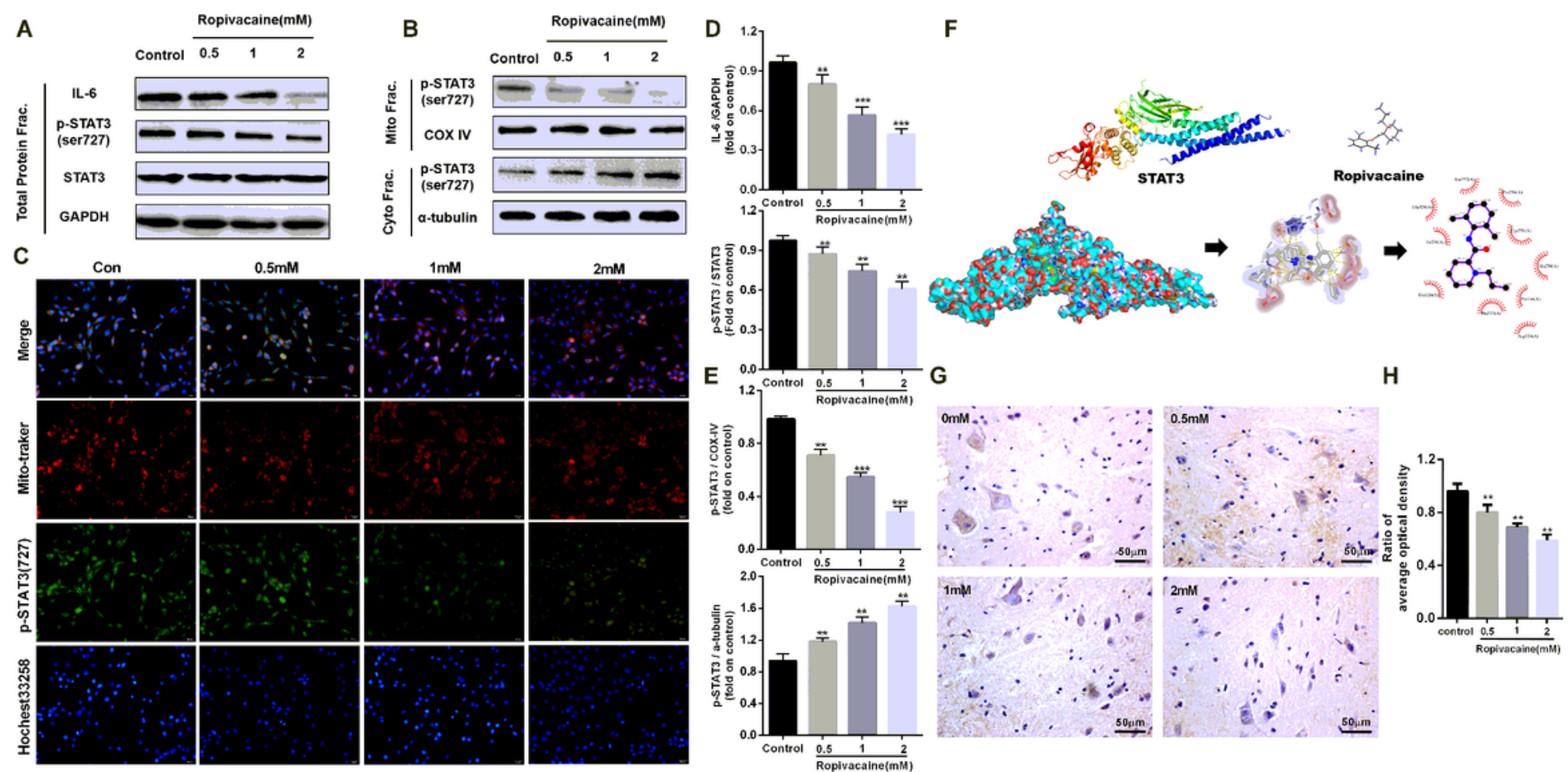

\section{Figure 6}

The effects of ropivacaine on the STAT3 signaling pathway. (A) PC12 cells were treated with different concentrations of ropivacaine. The protein expression of IL-6, STAT3, p-STAT3 (Ser727) and GAPDH in total protein were detected by Western-blot. (B) The protein expression of p-STAT3 (Ser727) in mitochondrial and cytoplasmic were detected by Western-blot. (C) Quantification of the gray values in graphs A and B. (D) Effect of ropivacaine on the colocalization of mitochondria (red) and STAT3(green) was detected by fluorescence microscope and represented as the overlapping fluorescence for the red and green channels, where the combined pixels appeared yellow. (E) Rats have received subarachnoid catheterization, and injected with different concentrations of ropivacaine, the expression of p-STAT3 (Ser727) in the spinal cord was measured by Immunohistochemistry. (F) Computational modeling of ropivacaine binding to the STAT3.The results are presented as the mean $\pm S D(n=3)$, where ** $p<0.01$, $\star \star \star ~ p<0.001$ compared with the control. 

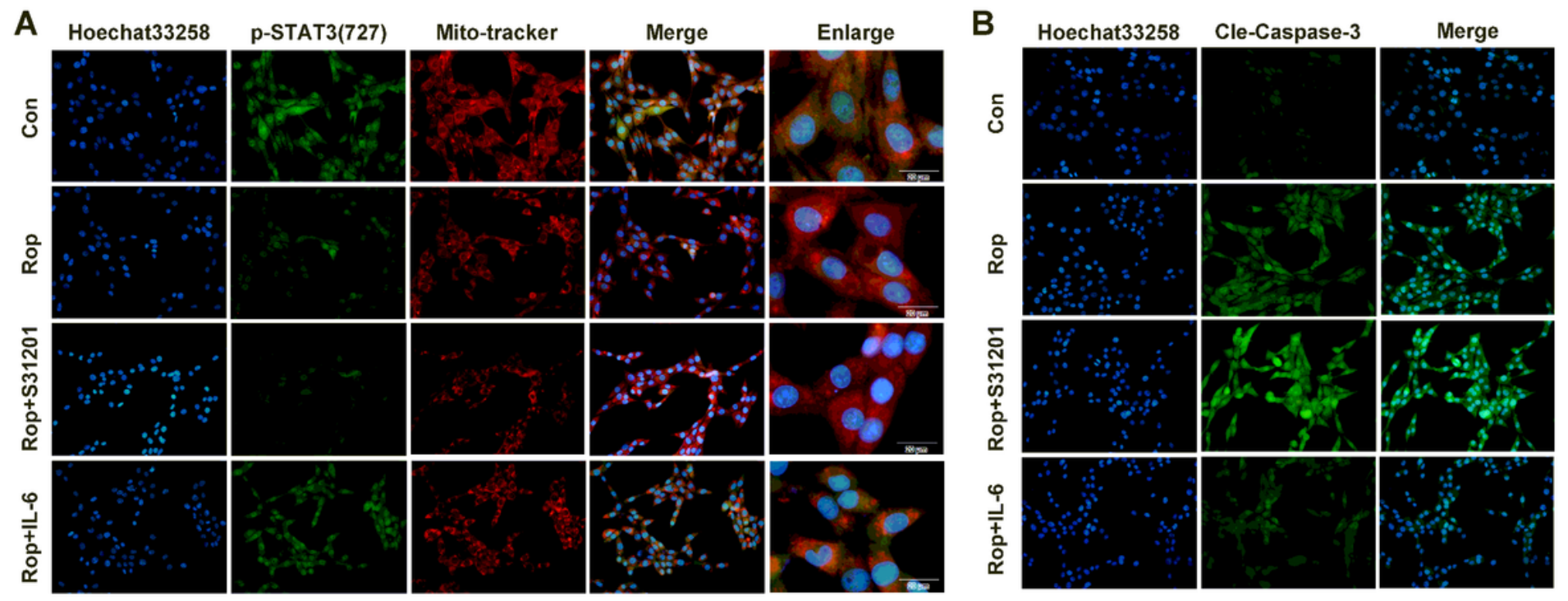

\section{Figure 7}

The effect of mitoSTAT3 on ropivacaine-induced apoptosis. A: PC12 cells were pre-treated with the inhibitor ( S31201) and activator (IL-6) for mitoSTAT3 for $2 \mathrm{~h}$, then adding $1 \mathrm{mM}$ ropivacaine to co-culture for 24h. the colocalization of mitochondria (red) and STAT3(green) was detected by fluorescence microscope and represented as the overlapping fluorescence for the red and green channels, where the combined pixels appeared yellow. (B) the expression of Cle-Caspase- 3 was detected by immunofluorescence. 